\title{
Surveying primary teachers about compulsory numeracy testing: combining factor analysis with Rasch analysis
}

Peter Grimbeek Griffith University

<p.grimbeek@griffith.edu.au>

\author{
Steven Nisbet Griffith University
}

<s.nisbet@griffith.edu.au>

\begin{abstract}
This paper reports the use of analytic methods, including Rasch item analysis, to re-examine teacher responses to questionnaire items probing opinions related to the compulsory numeracy tests conducted in Years 3, 5 and 7, in Queensland, Australia. Nisbet and Grimbeek (2004) previously reported an interpretable and statistically acceptable 6-factor exploratory factor solution. The present paper improved on this outcome by utilising Rasch analysis to identify items with orderly sequences of scores across response categories, and subjecting these to fresh exploratory and confirmatory factor analysis. The resulting 3-factor scale proved acceptable in terms of exploratory and confirmatory factor analysis and also in terms of Rasch item analysis. The paper briefly discusses the implications of these outcomes in terms of the refined instrument's capacity to gather information about how teachers view the Queensland numeracy reporting system.
\end{abstract}

\section{Keywords}

Rasch EPA CFA numeracy primary testing

\section{Introduction}

In the last 10 years, numeracy skills have been subject to much debate and scrutiny, with increased pressure being placed on primary schools to improve outcomes and report on progress. A review of the school curriculum (Wiltshire, McMeniman, \& Tolhurst, 1994) led to the introduction of the Year 2 Diagnostic Net and Year 6 Test in schools in the mid 1990s (Queensland Schools Curriculum Council, 1996). Although the Year 6 Test was discontinued in 1997 (making way for the federally-initiated Year 3, 5, \& 7 Tests), the Year 2 Net continues to be used. It has been received well by primary teachers and has had a positive impact on their teaching of mathematics (Nisbet \& Warren, 1999).

Furthermore, at a national level, performance-based assessment and reporting was promulgated in the mid 1990s (Australian Education Council, 1994a), and all states were given individual responsibility for the implementation of these procedures. Consequently in Queensland, Student Performance Standards (Australian Education Council, 1994b) were unsuccessfully introduced system-wide against teacher opposition despite substantial funds provided for professional development of teachers (Nisbet, Dole, \& Warren, 1997). Teachers rejected the increased workload imposed by the system and used the union to pressure the government to abandon the scheme.

In 1997, a National Literacy and Numeracy Plan was adopted in all states to (a) identify students at risk, (b) conduct intervention programs, (c) assess all students against national benchmarks, and (d) introduce a national numeracy reporting system (Department of Education, Training, \& Youth Affairs, 2000). Consequently, annual compulsory state-wide testing was introduced for students in Years 3, 5, and 7 in 1998. As a result, in August each 
year, all students in Years 3, 5, and 7 in Queensland government schools sit for tests in numeracy.

In Queensland tests, a broad interpretation of numeracy has been assumed, embracing the perspectives offered by Willis (1998) that numeracy (a) includes concepts, skills and processes in mathematics, (b) is described in terms of everyday situations in which mathematics is embedded; and (c) implies that students can choose and use mathematical skills as part of their strategic repertoire. Hence the Queensland tests cover number, measurement, geometry, chance, and data. They also test skills of calculation (written, mental \& calculator methods), and real-world problem solving.

A review of the Year 3, 5, and 7 testing program (Queensland School Curriculum Council, 1999) identified potential benefits and concerns related to such state-wide testing. The suggested benefits for teachers included the identification of student strengths and weaknesses, data to inform planning and teaching, the provision of results for various groups (boys, girls, students of non-English speaking backgrounds [NESB], indigenous students), and identification of teacher professional development needs. Issues of concern include the potential of the test to result in narrowing the curriculum, the testing regime may result in teachers teaching to the test, having assessment items not based on the classroom program, and the misuse of results (e.g., the publication of 'league tables' of 'good' and 'bad' schools).

The reports sent to schools after the annual tests are intended to provide administrators and teachers with information that will allow them to identify strengths and weaknesses of the school's program, compare their results with those of other schools, and to take what they may consider to be appropriate action. Information provided to the school includes results for each test item and each section (number, space, measurement and data) for each year-level, for each subgroup (boys, girls, NESB, \& indigenous students), and for each student, with comparisons with the state averages. Furthermore, the incorrect answers were recorded for each item for each student, and items for which the school scored 15\% above and 15\% below the state average was listed.

However, it is not known whether the schools' intentions to use the information provided in the results reflect the views of class teachers (and not just the principal) and whether schools and teachers actually put the test results to such uses. Evidence gathered in a pilot study suggests that, although schools might have good intentions, they don't necessarily carry them out and use the results for the benefit of their school's program or the students' performance levels. The previous study by Nisbet \& Grimbeek (2004) was designed to determine the extent to which schools analyse and use the test data and teachers' views of the validity or otherwise of the Year 3, 5 and 7 tests.

Traditional models of implementing innovation assume that teacher change is a simple linear process: Staff development activities lead to changes in teacher knowledge, beliefs and attitudes, which, in turn, lead to changes in classroom teaching practices, the outcome of which is improved student learning outcomes (Clarke \& Peter, 1993). Later models of teacher change regard teacher change as a long-term process (Fullan, 1982) with the most significant changes in teacher attitudes and beliefs occurring after teachers begin implementing a new practice successfully and see changes in learning (Guskey, 1985). The professional development (PD) models of Clarke (1988) and Clarke and Peter (1993) are refinements of the Guskey model that recognise the ongoing and cyclical nature of PD (focussing on knowledge, attitudes, and beliefs), and teacher change. 
Such models can help explain why some educational innovations are successful and others not. The introduction of the Year 2 Diagnostic Net was successful because teachers saw positive outcomes for pupils and because they valued the Net's overall effect (Nisbet \& Warren, 1999). However the introduction of Student Performance Standards in mathematics was a failure because teachers did not believe that the extra work entailed in performancebased assessment and reporting was worthwhile. Furthermore, they received little support for the move (Nisbet, Dole, \& Warren, 1997).

After five years of administration of the Years 3, 5, and 7 tests it was considered appropriate to investigate the impact of the tests on schools. Hence, the current dataset was collected (See Method section). The aim of the study was to investigate teacher attitudes to and beliefs about the Year 3, 5, and 7 tests (agreement with tests, their validity, and purposes), how school administrators (i.e., principals and deputy principals) and teachers use the test results (identifying students with difficulties, and gaps in the curriculum), the impact of the tests on teachers' practices (preparation for the test, influence on content and method), and the responses of teachers and pupils to the tests. A further aim was to determine the effect of school location, school size, experience, and extent of PD on such attitudes, beliefs and practices (Nisbet \& Grimbeek, 2004).

Responses to items revealed the following about teacher beliefs and attitudes:

- - Feedback: Only a minority of teachers give students feedback on strengths and weaknesses, or use the results to encourage pupils.

- - Diagnosis: A majority of teachers report that their school use the results to identify topics causing difficulties, identify gaps in content taught, and identify pupils experiencing difficulties.

- - Teacher change: A minority of teachers report that test results have influenced what they teach in mathematics lessons, and how they teach and assess it.

- - Comparison: Only a minority of teachers agree that the tests are a good way of comparing their school with other schools or with the state.

- - Validity: Most teachers think that the tests have little validity, in that these tests do not give an indication of numeracy ability, the quality of the school's numeracy program, or the teacher's ability to teach mathematics.

- - Preparation for tests: The vast majority of teachers report showing pupils how to fill in answers before the day of the test and giving pupils a practice test before the day of the actual test.

Nisbet and Grimbeek (2004) used exploratory factor analysis (EFA) procedures to examine the dataset. They reported that the 29 items were factorable (KMO $>0.800$ ), although the initial Principal Axis Factoring ${ }^{1}$ (PAF) and Varimax (orthogonal) rotation produced a sixfactor solution that was neither simple (some items loaded $>.30$ on more than one factor) nor interpretable (items did not group sensibly).

\footnotetext{
1 A method of extracting factors from the original correlation matrix, with squared multiple correlation coefficients placed in the diagonal as initial estimates of the communalities (See SPSS help files for more detail).
} 
Table 1. Six-factor solution for 15 items (PAF extraction, Varimax rotation, $\geqq 0.25$ loadings)

Items/factors
I use the results of the numeracy tests to give
students feedback on their strengths (Q19)
I use the results of the numeracy tests to
inform students about their weaknesses (Q20)
I use the results of the numeracy tests to
encourage students (Q21)

Our school analyses the results of the Year 3, 5, 7 numeracy tests after the results are received to identify gaps in content taught

(Q7)

Our school analyses the results of the Year 3, 5, 7 numeracy tests after the results are received to identify topics causing difficulty (Q8)

Our school analyses the results of the numeracy tests after the results are received to identify pupils with difficulties (Q6)

The numeracy tests have influenced how I teach in mathematics (Q28)

The numeracy tests have influenced how I assess the pupils in mathematics (Q29)

The numeracy tests have influenced what I teach in mathematics (Q27)

The numeracy tests are a good way of comparing our school with other schools (Q14)

The numeracy tests are a good way of comparing our school with the whole state (Q15)

The results of the Year 3, 5, 7 numeracy tests give an accurate indication of the teacher's ability to teach mathematics (Q2)

The results of the Year 3, 5, 7 numeracy tests give an accurate indication of the quality of the school's numeracy program (Q3)

Our school shows pupils how to fill in the answers on the tests before the day of the test (e.g., colouring bubbles, writing numbers in the boxes (Q4)

Our school gives pupils a practice numeracy test before the day of the test (Q5)

\subsection{3}

\subsection{3}

0.706 
After removal of items with loadings exceeding 0.30 on two or more factors or without significant loadings, a refined analysis with 15 of the 29 items resulted in a factorable $(\mathrm{KMO}=.766)$ six-factor solution that was both simple and highly interpretable. As shown in Table 1, the six factors could be labelled and described as follows (in order of factors):

- Feedback (three items): Teachers using the test results to encourage students, and to give them feedback on their strengths, and weaknesses.

- Diagnosis (three items): School using results for diagnostic purposes - to identify pupils with difficulties, identify gaps in content, and identify topics causing difficulties.

- Teacher change (three items): Tests influencing teacher practice in mathematics - what and how they teach it, and how they assess it.

- Comparison (two items): Tests as a good way of comparing the school with other schools and the state system.

- Validity (two items): The tests seen as valid indicators of the teachers' ability and the school's numeracy program.

- Preparation for tests (two items): Teachers showing pupils how to fill in answers, and giving practice tests.

Factor scores based on these clusters of items were used to examine the relationships between the six factors and specific background variables (geographical location, school size, teacher experience, and amount of PD). For instance, it was revealed that the factor 'teacher change' was affected by school size (teachers in smaller schools were influenced more in their teaching by the results of tests), and also the factor 'diagnosis' was affected by amount of PD (those with exposure to mathematics PD were more likely to use the tests to identify difficult topics, gaps in the curriculum and students experiencing difficulties).

The present paper set out to improve on this outcome by utilising Rasch analysis to identify items with orderly sequences of scores across response categories, and subject these to fresh exploratory and confirmatory factor analysis. The following statistical software was used to conduct the analyses: Statistical Package for the Social Sciences (SPSS) and Analysis of Moment Structures (AMOS), both available from http:/www.spss.com/, and WINSTEPS (Software for Rasch item analysis available from http://www.winsteps.com/). The rationale for so doing was that, regardless of the acceptability of the factor structure reported above, the process of identifying statistically acceptable items and subscales might be enhanced by combining judicious use of Rasch measurement data screening techniques available via WINSTEPS with the confirmatory factor analytic procedures afforded by AMOS. Insomuch as data analysts tend to use one or the other of these two analytic methods in isolation, this paper can claim some novelty value based on this 'mixed-method' approach to item and factor analysis. It's certainly the case that no other researcher has applied these methods to the instrument under scrutiny in this study.

\section{Methodology}

As reported by Nisbet and Grimbeek (2004), the dataset was collected by survey method ${ }^{2}$. A questionnaire was constructed containing items about teachers' attitudes, beliefs and practices relating to the state-wide Year 3, 5, and 7 Tests, plus items relating to the teachers' grade

2 Staff from Australian Council for Educational Research (ACER) provided assistance with the sample design and selected the sample of schools. The ACER sampling frame is compiled annually from data provided by the Commonwealth and each State and Territory education system. 
level, teaching experience, school location and school size, and an item for 'any other comments'. The results of a pilot study of 34 teachers in city and rural schools conducted in the previous months (Nisbet, 2003) were used to revise and expand the questionnaire items. A five-point Likert scale ('Disagree strongly' -> 'Agree strongly') was provided for responses, and teachers invited to comment on selected items. A sample of 56 primary schools representative of size, disadvantaged-schools index and geographical location across Queensland was selected and a total of 500 questionnaires sent to schools (having estimated the number of teachers in each school from the data on pupil numbers). Although the response rate (24\%) was small $(\mathrm{N}=121)$, the sample was representative in terms of year level and position (i.e., Years 1-7; principal, deputy, and mathematics coordinator), and in terms of teaching experience (1-40 years), geographical location (Capital city, provincial city, rural \& remote), and school size ( $<20$ pupils to $>400$ pupils).

SPSS was used as a first approximation of the frequencies per response category for Likert Scale items. It was also used to collapse response categories from five into four after using WINSTEPS to judge the ordering of response categories, and to conduct exploratory factor analyses.

Table 2: Example of disordered response categories identified by using WINSTEPS

\begin{tabular}{|l|c|c|}
\hline Item & Code (Response category) & Average score \\
\hline Q4 & 1 & -1.85 \\
\hline Q4 & 2 & -0.22 \\
\hline Q4 & 3 & -0.64 \\
\hline Q4 & 4 & -0.24 \\
\hline Q4 & 5 & -0.06 \\
\hline
\end{tabular}

WINSTEPS (3.5.3) was used to examine item statistics related to the ordering of Likert scale response categories across the 29 items (Ref., Bond \& Fox, 2001). Table 2 illustrates an item in which the average score for the 'disagree' (code 2) was more positive than for the 'undecided' (Code 3) or 'Agree' (Code 4) response categories. It appears that participants found it as easy to tick 'disagree' as 'strongly agree' for this item, and found it more difficult to tick 'undecided' or 'agree', whereas normally one would expect the 'agree' response to be more difficult to tick than 'undecided' or 'disagree'. Based on such an examination, the two upmost-response categories of the 5-point scale were collapsed to form a 4-point scale. A subset of five items that continued to display disorderly response categories were excluded at this point, and the remaining items re-entered in an iterative sequence of exploratory (Principal Axis, Varimax rotation) and confirmatory (AMOS CFA) factor analyses. Finally, WINSTEPS was utilised to re-examine both the difficulty level and level of fit (via Infit and Outfit statistics) for items forming part of the refined factor structure.

A fundamental issue with the use of Likert scale items is the problematic measurement properties of multi-choice response categories per item. Differing assumptions about measurement properties determine conflicting rules of thumb for analysing such data (Grimbeek, Bryer, Beamish, \& D’Netto, 2005). A reason for resorting to Rasch analysis in this paper is that it explicitly takes into account the categorical or ordinal nature of such variables (ref., Michell, 1999, Bond \& Fox, 2001, Byrne, 2001). In contrast, the exploratory and confirmatory techniques also reported in this paper assume that the data to be analysed is implicitly parametric (equal interval, ratio) in nature. The implausibility of this assumption in 
relation to Likert scale items does not deter researchers from using parametric tools. A rule of thumb for such work is to assume that scales with 4 or more points approximate the properties of interval measures (Byrne, 2001, p.91-92).

SPSS factor analysis was used to conduct fresh exploratory factor analyses (PAF extraction, Varimax rotation) on the 24 items remaining after using WINSTEPS to identify and exclude five items with persistent disorderly response categories. Subsequently, AMOS 5.01 was used to undertake confirmatory factor analysis (CFA) on the items and factors identified via the EFA procedure outlined previously. A reason for using the CFA as well as EFA (i.e., PAF, Varimax rotation) procedure is that whereas EFA makes no assumption about item-scale associations, CFA explicitly tests the proposition that items cluster in specific subscales and take it further. Its rigorous testing procedures include a suite of fit estimates, ranging from Chi Square statistics through various types of model fit (see below) that permit a more rigorous scrutiny of outcomes than is afforded via EFA.

Table 3: Estimates of goodness of fit for the 15-item and 7-item CFA models

\begin{tabular}{lccc}
\hline Measure & 15-item model & 7-item model & Chi-Square difference test \\
\hline Chi square & 87.079 & 12.165 & 74.914 \\
df & 75 & 11 & 64 \\
Probability & $\mathbf{0 . 1 6 1}$ & $\mathbf{0 . 3 5 1}$ & 0.165 \\
Chi/Df & $\mathbf{1 . 1 6 1}$ & $\mathbf{1 . 1 0 6}$ & \\
RMR & $\mathbf{0 . 0 4 8}$ & $\mathbf{0 . 0 2 5}$ & \\
RMSEA & $\mathbf{0 . 0 3 7}$ & $\mathbf{0 . 0 3}$ & \\
NFI & $\mathbf{0 . 9 2 7}$ & $\mathbf{0 . 9 7 9}$ & \\
RFI & 0.897 & $\mathbf{0 . 9 6 0}$ & \\
TLI & $\mathbf{0 . 9 8 4}$ & $\mathbf{0 . 9 9 6}$ & \\
CFI & $\mathbf{0 . 9 8 9}$ & $\mathbf{0 . 9 9 8}$ & $\mathbf{0 . 9 7 2}$ \\
GFI & $\mathbf{0 . 9 1 7}$ & $\mathbf{0 . 9 2 8}$ & \\
AGFI & 0.868 & &
\end{tabular}

\section{Results}

WINSTEPS-based examination of the 29 items indicated that the average response was out of sequence across response categories for 13 of the 29 items. Of these items with disordered categories, nine involved the top-most category, usually because of sparse selection of this response option. Accordingly, the two topmost categories (representing Agreement \& Strong agreement) were collapsed to form a 4-point scale. On examination, it was found that the average response remained out of sequence for five items in this 4-point scale. While more radical collapsing into trichotomous or dichotomous response categories would undoubtedly 
have minimised the number of out of order sequenced response categories, doing so would have infringed conventional rules of thumb regarding the use of such survey items in exploratory or confirmatory factor analyses. That is, as stated above, 4-point response scales are regarded as at the lower limits of acceptability (Byrne, 2001) for factor analysis.

The initial 6-factor solution was further examined by confirmatory factor analysis (CFA). Given the very low level of missing data ( 1 case in each of 9 items, 2 cases in the $10^{\text {th }}$ ), SPSS Replace Missing Values was used to replace these with the average for that item. As indicated in Table 3, estimates of goodness of fit for the initial 15-item, 6-factor model either exceeded or approximated acceptable thresholds.

That is, the chi-square/degrees of freedom computation (correcting chi square for model complexity) approximated the acceptable $0-3$ range; estimates of residual variance (root mean square residual [RMR], root mean square error of approximation [RMSEA] approximated the acceptable 0-.05 range; and model comparisons with the baseline independence model (normed fit index [NFI], Tucker-Lewis index [TLI], comparative fit index [CFI], the relative fit index [RFI], and a subset of other goodness-of-fit estimates (goodness of fit index [GFI], adjusted goodness of fit index [AGFI] approximated the acceptable 0.9-1.0 range or were marginal to this range. In short, as shown in Table 3, above, structural equation modelling (AMOS) associated with the initial 6-factor model indicated the model to be highly acceptable in statistical as well as conceptual terms.

Table 4. Three-factor solution for 7 items (PAF extraction, Varimax rotation, $\geqq 0.25$ loadings)

Factors/Items

1

2

3

Our school analyses the results of the Year 3, 5, \& 7 numeracy tests after the results are received to identify topics causing difficulties

(Q8)

Our school analyses the results of the Year 3, 5, \& 7 numeracy tests after the results are received to identify gaps in content taught (Q7)

Our school analyses the results of the numeracy tests after the results are received to identify pupils with difficulties (Q6)

0.781

I use the results of the numeracy tests to give students feedback on their strengths (Q19)

I use the results of the numeracy tests to inform students about their weaknesses (Q20)

The results of the Year 3, 5, \& 7 numeracy tests give an accurate indication of the quality of the school's numeracy program (Q3)

The results of the Year 3, 5, \& 7 numeracy tests give an accurate indication of the teacher's ability to teach mathematics (Q2)

The 6-factor model dataset was subjected to an iterative sequence of confirmatory factor analyses (including the exclusion of six cases classified as extreme examples of multivariate kurtosis - Mahalanobis estimates) that resulted in the 7-item, 3-factor model illustrated in Table 4 in terms of EFA style output. This three-factor solution included Diagnosis (3 items), Feedback (2 items), and Validity (2 items) factors present in the initial analysis but excluded seven items related to Teacher change (3 items), Comparison (2 items), and Preparation for testing (2 items). 
From a confirmatory factor analytic perspective, the revised 7-item, 3-factor model achieved a high standard. As shown in Table 3, all 10 listed estimates of goodness of fit achieved highly acceptable levels.

The two models were compared by using chi-square values to compute the chi-square difference test. This test examines the significance of the chi-value obtained by taking into account the difference in chi values and the difference in degrees of freedom. As indicated in Table 3, the two models appear statistically equivalent in terms of this test.

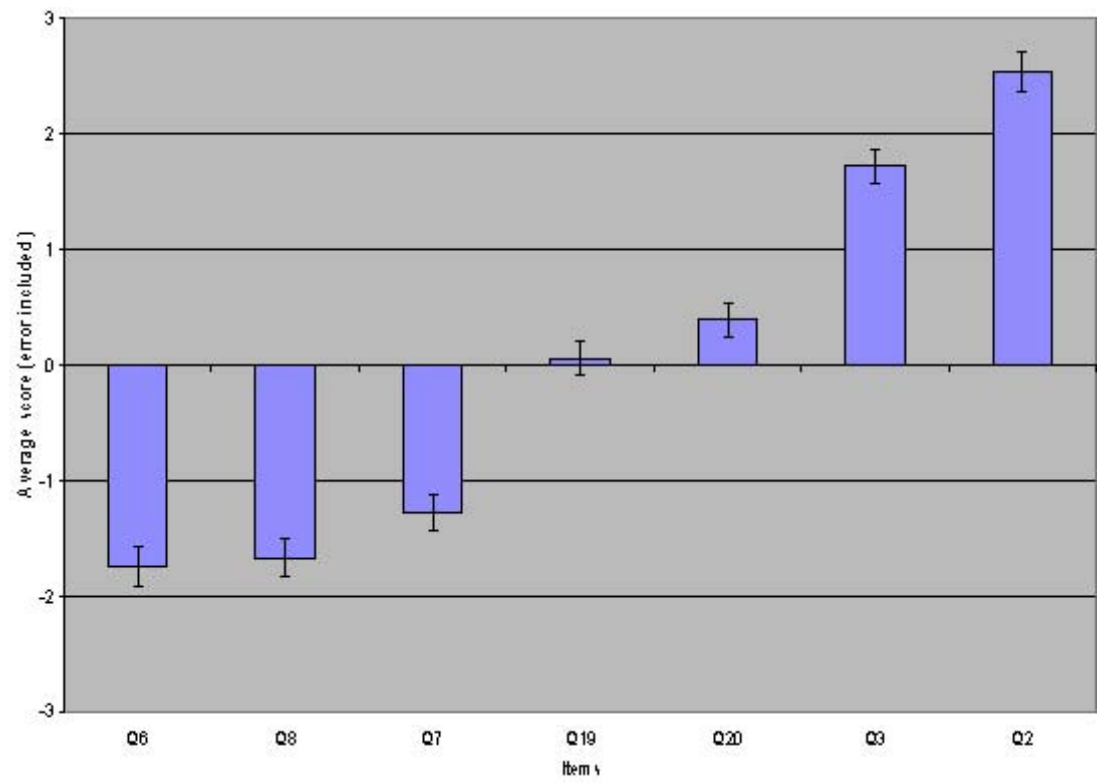

Figure 1. Illustration of average score per measure

Finally, WINSTEPS was used to examine the average scores per item and response category. Consistent with the prior selection process (Figure 1), the average response was in sequence across response categories for all seven items. That is, the average estimates of level of agreement per item clustered consistently with the three subscale factor structure.

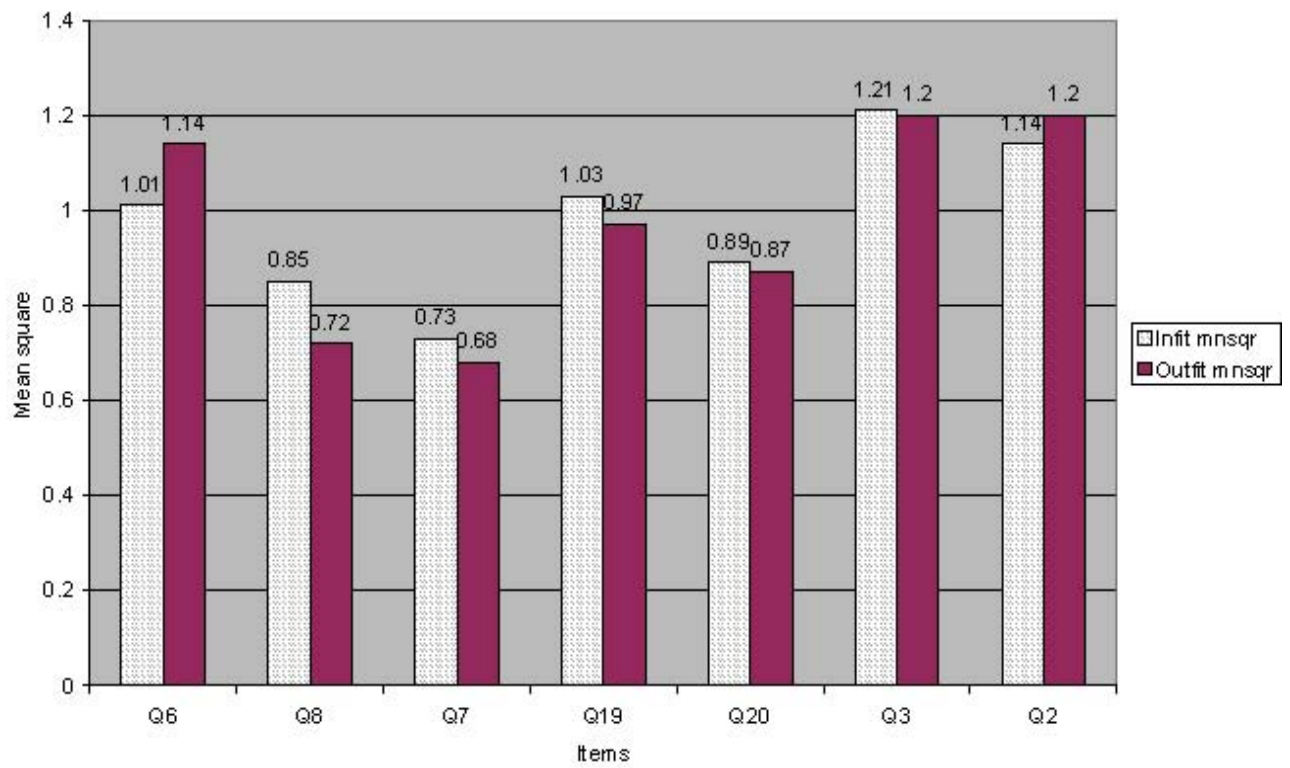

Figure 2. Illustration of Infit plus Outfit measures per item 
In addition (Figure 2), the mean square fit values computed for measures of Infit and Outfit per item all occupied the 0.5-1.7 bandwidth of values considered consistent with these items being neither too easy nor too difficult (Smith, Schumacker, \& Bush, 1998; Wright \& Linacre, 1994). In other words, in the context of response categories based on level of agreement, none of these items attracted $100 \%$ of responses for "Strongly agree" (i.e., "too easy" would be equivalent to all participants strongly agreeing) or "Strongly disagree" (i.e., "too difficult” would be equivalent to all participants strongly disagreeing).

\section{Discussion and conclusions}

The rationale for the present study was that combining Rasch item analysis with exploratory and confirmatory factor analysis would optimise the resulting factor solution. As it turns out, the initial 15-item solution could be replaced by a 7-item model with highly acceptable statistical criteria, including those based on Rasch item analysis.

From the point of view of sampling teacher opinions related to mathematics testing, the optimised instrument offers increased ease of use in that the diminished number of items is more readily administered via the web-based surveys in vogue at present. The statistical equivalence of these two models, as per the chi-square difference test, places the two models on a level footing, but the 3-factor model is certainly more parsimonious as well as also being easier to analyse. More generally, the outcomes reported here suggest the importance and pertinence of items related to Diagnosis (3 items), Feedback (2 items), and Validity (2 items) when canvassing teacher views related to numeracy education.

As a bonus, the three subscales associated with the seven items range neatly along a single scale in terms of item difficulty (Figure 1). That is, from a Rasch item analysis perspective, the seven items could be viewed as forming a single scale that collects information across a set of seven items where the responses vary in terms of level of agreement. This interpretation of the three subscales is supported by the outcomes of a single factor PAF (details not reported here) indicating that all seven items load significantly on a single scale and could be administered and scored as such. The benefit to the test administrator is that responses to these seven items can either be scored in terms of three more specific factors (Diagnosis, Feedback, and Validity) or as a single 7-item measure indicative of the positivity of teacher attitudes concerning the Year 3, 5, 7 tests of numerical literacy.

This is not to say that the instrument has reached its academic destination ${ }^{3}$ in terms of refining items to minimise ambiguity, enhancing the reliability of outcomes by generating additional items, and enhancing the validity of outcomes by ensuring that the set of items is systematically extended in terms of the measurement of factors of interest. In all of this, we'd expect the combination of Rasch item analysis and confirmatory factor analysis to continue to provide highly useful information.

In these terms, a criticism of the revised factor structure is that it retains approximately $1 / 4$ of the initial set of 29 items. As stated above, this trimmed set of items is highly acceptable from a variety of statistical perspectives (exploratory factor analysis, confirmatory factor analysis, Rasch item analysis), but the loss of $3 / 4$ of the items does diminish the statistical reliability of judgments based on the total number of items. It also diminishes validity insomuch as these rigorously applied statistical criteria enhance the purity of the measure but diminish the

\footnotetext{
${ }^{3}$ For example, some of the items could be rewritten more succinctly.
} 
breadth of the field from which items are drawn. Anecdotally, as a research methodologist in contact with Australian academics, the first author can report that several seasoned researchers have rejected Rasch item analysis as a legitimate procedure precisely because of the tension between the development of such pure but sparse measures and the pressing need to develop measures with ecologically reliable and valid qualities.

It follows that potential test users in the field (e.g., mathematic educators) might consider such sparse measures to lack practical application but the authors are of the opinion that practitioners could use this test to identify responses relevant to the three remaining factors diagnosis, feedback and validity. While the revised factor structure omits three of the six initial factors (i.e., teacher change, comparison, and preparation were omitted), the three remaining factors (diagnosis, feedback, and validity) are crucial to the process of monitoring teacher beliefs about the numeracy tests.

As stated above, a major advantage of the trimmed factor structure is that it not only clarifies the measures but also reduces the length of the questionnaire, and in doing so makes the exercise of gathering teacher opinions about the compulsory Year 3, 5, and 7 numeracy tests easier and thus more likely to result in higher response rates. In short, insomuch as this instrument was designed to determine the extent to which schools analyse and use the test data, and to probe teacher views of Year 3, 5, and 7 tests, this revision meets that need very well.

Finally, it is clear that the application to the dataset of Rasch item analysis together with confirmatory factor analysis has produced an instrument with a factor structure that is statistically and possibly conceptually elegant by comparison with the model initially reported, and could be used to survey teacher views related to numeracy education testing as either a three- scale or single-scale instrument.

\section{References}

Australian Education Council (1994a). Mathematics work samples. Carlton, Vic: Curriculum Corporation.

Australian Education Council (1994b). Student performance standards in mathematics for Queensland schools. Carlton, Vic: Curriculum Corporation.

Bond, T., and Fox, C. (2001). Applying the Rasch model: Fundamental measurement in the human sciences. USA: Lawrence Erlbaum Associates, Inc.

Byrne, B. M. (2001). Structural equation modeling with AMOS. London: Lawrence Erlbaum Associates, Publishers.

Clarke, D. J., \& Peter, A. (1993). Modelling teacher change. In B. Atweh, C. Kanes, M. Carss, \& G. Booker (Eds.), Contexts in Mathematics Education. Proceedings of the Sixteenth Annual Conference of the Mathematics Education Research Group of Australasia (MERGA), Brisbane, July 9-13, 1993. Brisbane: MERGA, 167-175.

Clarke, D. J. (1988). Realistic assessment. In D. Firth (Ed.), Maths counts - who cares? Parkville, Victoria: Mathematical Association of Victoria, 187-192.

Department of Education, Training \& Youth Affairs (2000). Numeracy, a priority for all. Canberra, ACT: Commonwealth of Australia.

Fullan, M. (1982). The meaning of educational change. New York: Teachers College Press. 
Grimbeek, P., Bryer, F., Beamish, W., \& D’Netto, M. (2005). Use of data collapsing strategies to identify latent variables in questionnaire data: Strategic management of Junior and Middle School data on the CHP questionnaire. Paper presented and published as part of Proceedings of the $3^{\text {rd }}$. Annual International Conference on Cognition, Language and Special Education, Gold Coast, Qld. Australia.

Guskey, T. (1985). Staff development and teacher change. Educational Leadership, 42, 5760.

Michell, J. (1999). Measurement in Psychology: A critical history of a methodological concept. United Kingdom: Cambridge University Press.

Nisbet, S., \& Grimbeek, P. (2004). Primary teachers' beliefs and practices with respect to compulsory numeracy testing. Mathematics education for the third millennium: Towards 2010. Townsville, Australia.

Nisbet, S., \& Warren, E. (1999). The effects of a diagnostic assessment system on the teaching of mathematics in the primary school. In O. Zaslavsky (Ed.), Proceedings of the $23^{\text {rd }}$ Annual Conference of the International Group for the Psychology of Mathematics Education. Haifa, Israel, July 25-30, 1999. Vol. 3, pp. 337-344.

Nisbet, S., Dole, S. \& Warren, E. (1997). Cooperative professional development for mathematics teachers: A case study. In F. Biddulph \& K. Carr (Eds.), People in Mathematics Education: Proceedings of the 20th Annual Conference of Mathematics Education Research Group of Australasia (Rotorua, July 1997). Hamilton, Waikato, NZ: MERGA.

Queensland School Curriculum Council (1999). Review of Queensland Literacy and Numeracy Testing Programs, 1995-1999: Issues Paper. Brisbane: Queensland School Curriculum Council.

Queensland Schools Curriculum Council (1996). The Year 2 Diagnostic Net Handbook for Schools - Shaping the Future. Brisbane: The Queensland Government Press.

Smith, R.M., Schumacker, R.E., \& Bush, M. (1998). Using item mean squares to evaluate fit to the Rasch model. Journal of Outcome Measurement, 2, 66-78.

Willis, S. (1998). Which numeracy? Unicorn, 24, 32-42.

Wiltshire, K., McMeniman, M., \& Tolhurst, T. (1994). Shaping the Future: Review of the Queensland School Curriculum. Brisbane: Queensland Legislative Assembly.

Wright, B. D., \& Linacre, J. M. (1994). Reasonable mean-square fit values. Rasch Measurement Transactions, 6, 205. 